\title{
A Topological Data Analysis Perspective on Non-Covalent Interactions in Relativistic Calculations
}

\section{Małgorzata Olejniczak $^{1}$} Julien Tierny ${ }^{3}$

\footnotetext{
${ }^{1}$ Centre of New Technologies, University of Warsaw, S. Banacha 2c, 02-097 Warsaw, Poland

${ }^{2}$ Université de Lille, CNRS, UMR 8523 PhLAM - Physique des Lasers, Atomes et Molécules, F-59000 Lille, France

${ }^{3}$ Sorbonne Université, CNRS, Laboratoire d'Informatique de Paris 6, LIP6, F-75005 Paris, France
}

\section{Correspondence}

Małgorzata Olejniczak, Centre of New Technologies, University of Warsaw, S. Banacha 2c, 02-097 Warsaw, Poland Email: m.olejniczak@cent.uw.edu.pl

\section{Funding information}

Polish National Science Centre (2016/23/D/ST4/03217), Labex CaPPA (ANR-11-LABX-0005-01) and I-SITE ULNE project OVERSEE (ANR-16-IDEX-0004), CPER CLIMIBIO (European Regional Development Fund, Hauts de France council, French Ministry of Higher Education and Research), the French national supercomputing facilities (DARI A0050801859), the European Commission grant H2020-FETHPC-2017 “VESTEC” (ref. 800904)

\section{André Severo Pereira Gomes ${ }^{2}$}

Topological Data Analysis (TDA) is a powerful mathematical theory, largely unexplored in theoretical chemistry. In this work we demonstrate how TDA provides new insights into topological features of electron densities and reduced density gradients, by investigating the effects of relativity on the bonding of the $\mathrm{Au}_{4}-\mathrm{S}-\mathrm{C}_{6} \mathrm{H}_{4}-\mathrm{S}^{\prime}-\mathrm{Au}_{4}{ }_{4}$ molecule. Whereas recent analyses of this species carried out with the Quantum Theory of Atoms-In-Molecules [Anderson et al., Chem. Eur. J. 25, 2538, 2019] concluded, from the emergence of new topological features in the electron density, that relativistic effects yielded non-covalent interactions between gold and hydrogen atoms, we show from their low persistence values (which decrease with increased basis set size) these features are not significant. Further analysis of the reduced density gradient confirms no relativity-induced noncovalent interactions in $\mathrm{Au}_{4}-\mathrm{S}-\mathrm{C}_{6} \mathrm{H}_{4}-\mathrm{S}^{\prime}-\mathrm{Au}_{4}$. We argue TDA should be integrated into electronic structure analysis methods, and be considered as a basis for the development of new topology-based approaches.

Keywords - noncovalent interactions, relativistic effects, quantum chemistry, topological data analysis. 


\section{1 | INTRODUCTION}

A well-exploited consequence of the Hohenberg-Kohn theorem [4] is that the exact, non-degenerate, ground-state electron density (ED) contains complete molecular information and therefore encodes all interactions in molecular systems. In order to retrieve information on interatomic interactions from the ED, this property is subjected to various analysis schemes, which often involve visual exploration. This is, however, both a technical challenge and a test of chemical concepts. The former relates to a nontrivial choice of analysis techniques of scientific data. For instance, direct visualization schemes (an example of which is representing 3D scalar fields as isosurfaces), although intuitive and simple in realization, often lead to images that are cluttered and difficult to grasp, especially when aimed to illustrate complex scientific questions. On the other hand, the so-called feature-based visualization methods are designed to recapture only the meaningful features of data, however are more complex to comprehend and therefore not as widely used. [48. 87]

Topological Data Analysis is a recent research field, at the interface between mathematics and computer science, which suggests to investigate data based on its structure. 41 Rooted in sound theoretical settings such as Morse theory, 52] TDA provides to users a set of tools for the robust extraction of topological features in their data. This includes for instance critical points, integral lines, separating surfaces, voids, etc. Moreover, Persistent Homology $\mathrm{PH}$ 31 brings an appealing framework for measuring the salience of topological features in the data, which is well established both at a theoretical and practical level.[55] In practice, it allows users to discriminate important features from non-significant configurations and provides the necessary basis for multi-scale data analysis. 85] Recent years have witnessed some successful applications of TDA to chemistry, where it helped to understand hydrogen-bonding networks in ion and molecule aggregates,[100, 101 81] aqueous solubility of molecules, [99 61] stability of fullerenes,[102] molecular transition pathways, [14] conformational spaces of molecules [50] or the bonding patterns in molecular systems. 95 38 $38.42 \quad 15$

In this manuscript we focus on the use of TDA to investigate non-covalent interactions ( $\mathrm{NCls}$ ) for systems containing heavy elements. The description of $\mathrm{NCls}$ is by itself a challenge for existing chemistry concepts, and has been a driving force for the development of new analysis techniques.[56 22] For systems containing heavy elements, there is the additional challenge of including the effects of relativity in the electronic structure as these can qualitatively change the properties of a molecular system. 69.78 79 27.70] The bonding analysis in such systems is especially demanding if the spin-orbit coupling $(\mathrm{SOC})$ is non-negligible. 26] There is currently intense activity in the development and practical use of new analysis tools and methods that can reliably characterize chemical bonding irrespective of the strength of SOC, and as we hope to show, TDA is a promising approach.

In order to illustrate and further extend the idea of using TDA for interatomic interactions, we have chosen a molecular system studied recently in ref.[7], $\mathrm{Au}_{4}-\mathrm{S}_{-} \mathrm{C}_{6} \mathrm{H}_{4}-\mathrm{S}^{\prime}-\mathrm{Au}_{4}{ }_{4}$ 1], which is interesting in the context of goldsulfur $39,58,103,72,73$ and gold-hydrogen $71,83,76,77,93$, interactions due to their ubiquity in metalorganic complexes and on interface - a proof of many faces of the fascinating chemistry of gold. [18 66 67, 68] A remarkable finding of the study of Anderson and co-workers $[7$ is that it characterized the emergence of $\mathrm{NCls}$ between gold and hydrogen centers, using the Quantum Theory of Atoms-In-Molecules (QTAIM). 10. 11

QTAIM is a very popular analysis tool,[64] which describes the stationary points of the ED gradient and interprets these points as chemical objects - maxima correspond to nuclei (although some molecules exhibit non-nuclear attractors 36]), while saddle points are identified as bond critical points (BCP), ring critical points (RCP) or cage critical points (CCP). ED gradient paths emanated from a saddle point, terminating at two maxima and traced along maximum ED, originally named bond paths, [9] are interpreted as interaction lines between atoms and the areas of space delimited by zero-flux surfaces of ED gradient define atomic basins. However, recent ambiguous results raised a discussion on the

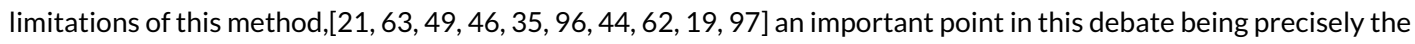


failure of QTAIM to study $\mathrm{NCl}$.

While it can be particularly appealing to use QTAIM in this context, especially due to its availability in many standard quantum chemistry codes and supposedly simple interpretation of topological features of ED, it is known that the latter is not a sensitive descriptor of subtle interatomic interactions, due to its exponentially decaying behavior away from the nuclei. This observation has led to the development of other descriptors, better suited for studying non-covalent interactions, such as the $\mathrm{NCl}$ index $45,24.54,53$ based on the reduced density gradient (RDG), $s(\vec{r})$ :

$$
s(\vec{r})=\frac{1}{2\left(3 \pi^{2}\right)^{1 / 3}} \frac{|\nabla \rho(\vec{r})|}{\rho(\vec{r})^{4 / 3}} .
$$

At critical points of ED where $\nabla \rho(\vec{r})=0$, RDG reaches its minimum value, $s(\vec{r})=0$, therefore tracing the minima of $s(\vec{r})$ can be used to locate the interaction sites in molecular systems. The minima of $s(\vec{r})$ may also appear in areas not associated with QTAIM critical points - referred to as "non-AIM critical points" or "interaction critical points (ICPs)"[23] 17] in which case they indicate very weak interactions.

In practical applications, both ED and RDG are approximate and their quality depends on the quantum mechanical (QM) model adopted in calculations. The approximations introduced with this model, 40, 65, 74 involving the choice of the Hamiltonian (determining whether and which relativistic effects are included), the method (responsible for the description of electron correlation) and the basis set (establishing the quality of one-electron functions), as well as other numerical parameters (such as the numerical thresholds, grids), can be assessed in a systematic way, yet there are relatively few studies illustrating how all of them affect the aforementioned fields and their topological features. $32,60,20,6$

Inaccuracies of QTAIM results also stem from theoretical and semantic reasons. The former is related to the fact that QTAIM uses concepts from the graph theory, hence inherits its limitations, in particular it can give the "static" or "binary" information about topological features of ED, but not on their evolution, stability or importance (contrary to TDA). The latter reason is a source of continuous debate, related to the misinterpretation of QTAIM concepts (an example of which is a confusion between "bond paths" and "chemical bonds" 12.80 62, 25]), and to the overuse of the adjective "topological" in chemistry which should not be confused with mathematical topology. [8] 51

The TDA approach to the interatomic interactions is more general. TDA provides information on the topological features of a scalar field, including their properties, configuration, evolution and significance, while staying oblivious to what the scalar field represents. This detachment of a tool and an interpretation of its output is very helpful to gain a new perspective on the research problem or to redefine existing concepts in a more robust way. In particular, TDA applied to ED, RDG and a signed electron density $\left(\tilde{\rho}(\vec{r})=\operatorname{sign}\left(\lambda_{2}(\vec{r})\right) \rho(\vec{r})\right.$ with $\lambda_{2}$ being the second eigenvalue of the ED Hessian) 24 enabled to automatically extract and classify interatomic interactions merely based on the topological persistence (vide infra) of critical point pairs of these fields. 38

\section{2 | COMPUTATIONAL DETAILS}

Following ref.[7], the geometry of the $\mathrm{Au}_{4}-\mathrm{S}-\mathrm{C}_{6} \mathrm{H}_{4}-\mathrm{S}^{\prime}-\mathrm{Au}_{4}$ molecule was optimized in the ADF software 84, 13 , 34] using three different models: (i) scalar-relativistic Zeroth-Order Regular Approximation (sr-ZORA), 90, 89. 91. 92 PBE functional[59] and TZP basis set [88] at the singlet spin-restricted level (denoted throughout the text as g1), (ii) sr-ZORA, PBE, TZP at the triplet spin-unrestricted level (g2) and (iii) sr-ZORA, PBE, QZ4P[88] at the triplet spin-unrestricted level with tighter SCF convergence criteria (g3). The study of a triplet state of this molecule, motivated by its proximity to the singlet ground state, was conducted to explore how the spin state affects the ED topology. 

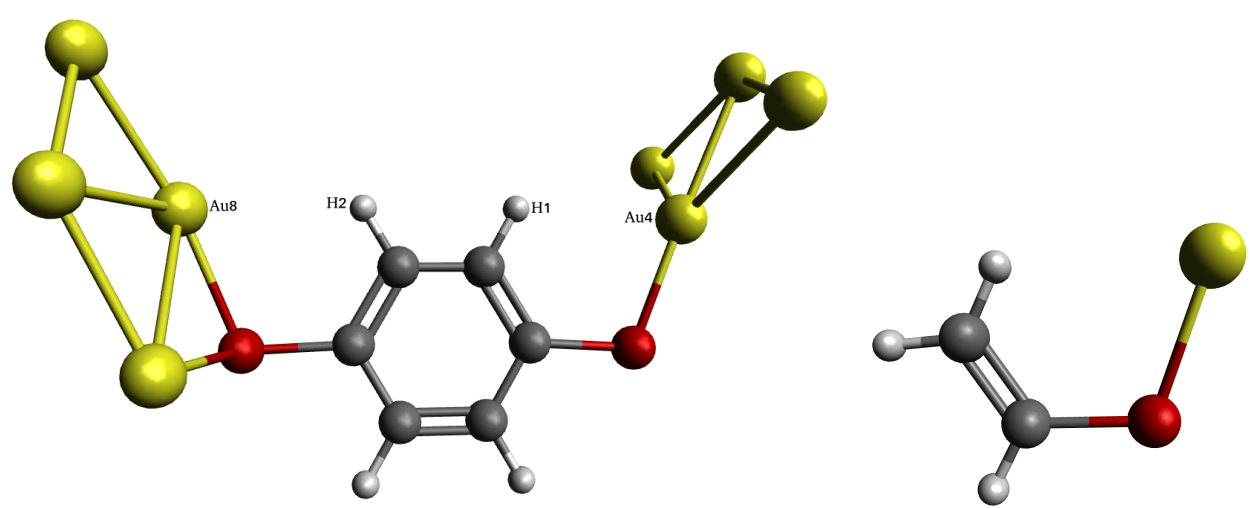

FIG URE 1 Molecular systems under study: $\mathrm{Au}_{4}-\mathrm{S}_{-}-\mathrm{C}_{6} \mathrm{H}_{4}-\mathrm{S}^{\prime}-\mathrm{Au}_{4}$ (left) and $\mathrm{Au}-\mathrm{S}-\mathrm{CH}-\mathrm{CH}_{2}$ (right). The numbering of selected $\mathrm{Au}$ and $\mathrm{H}$ centers is shown and is the same as in ref.[7]. Color coding for atoms: Au - yellow, S - red, C - dark gray, $\mathrm{H}$ - light gray. Additional information is available in ESI.

We considered simpler model systems of that molecule to investigate the importance of a local environment to the topological features of interest, $\mathrm{Au}-\mathrm{S}-\mathrm{CH}-\mathrm{CH}_{2}$ 1. Its geometry was obtained (without reoptimization) by removing atoms from the $\mathrm{g} 1$ model of $\mathrm{Au}_{4}-\mathrm{S}-\mathrm{C}_{6} \mathrm{H}_{4}-\mathrm{S}^{\prime}-\mathrm{Au}_{4}$ molecule, so that it contains only one gold atom linked by sulfur to the ethene group that replaced the benzene ring. As the positions of all nuclei in Au-S-CH-CH were preserved as in the original molecule and due to the lack of symmetry in $\mathrm{Au}_{4}-\mathrm{S}-\mathrm{C}_{6} \mathrm{H}_{4}-\mathrm{S}^{\prime}-\mathrm{Au}_{4}$, this simplification resulted in two geometries of the small model (atom numbers in parenthesis, after ref.7]) - $\mathrm{Au}(4)-\mathrm{S}(1)-\mathrm{C}(1) \mathrm{H}-\mathrm{C}(2) \mathrm{H}(1) \mathrm{H}($ "a") and $\mathrm{Au}(8)-\mathrm{S}(2)-\mathrm{C}(4) \mathrm{H}-\mathrm{C}(3) \mathrm{H}(2) \mathrm{H}$ ("b"), hence the nomenclature m-a-g1 and $\mathrm{m}-\mathrm{b}-\mathrm{g} 1$, respectively.

For all studied molecules, ED has been evaluated in single-point calculations with the ADF software. These employed the non-relativistic (NR) Hamiltonian as well as scalar and spin-orbit ZORA Hamiltonians (sr-ZORA and so-ZORA, respectively), DFT with different exchange-correlation (XC) functionals (PBE, B3LYP, 82, SAOP, 75, 37] M06-2X 105 104]) and three basis sets (DZ, 88 TZP and QZ4P). Additionally, for the small Au model we performed calculations in the locally-modified version of the DIRAC software 3] with the Lévy-Leblond (LL), 47] spinfree DiracCoulomb (SFDC) 28 and Dirac-Coulomb (DC) 94 Hamiltonians, two methods involving different levels of electron

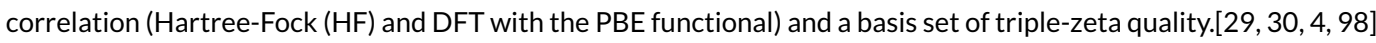

The TDA of the logarithms of ED and RDG $(\log (\rho)$ and $\log (s)$, respectively 1) was performed with the TTK software package. 86 All details can be found in the electronic supplementary information (ESI).

\section{3 | RESULTS AND DISCUSSION}

Even though the optimized geometries of the Au complex are slightly different than in ref.[7], our analysis confirms the main conclusions of the cited work: additional pairs of critical points of ED (1-saddles and 2-saddles, interpreted as "RCPs" and "BCPs", respectively) between Au and $\mathrm{H}$ nuclei appear for certain combinations of Hamiltonians, methods, basis sets, molecular geometries and spin states, but they never appear in the non-relativistic context.

The comparison of topological features of relativistic (so-ZORA) and non-relativistic EDs of g1 molecule obtained with the PBE/QZ4P QM model is illustrated in 2 where, in particular, the topological skeleton of non-relativistic ED (without these additional saddle-saddle pairs) and relativistic ED (with these pairs) are shown. The results from TDA of 

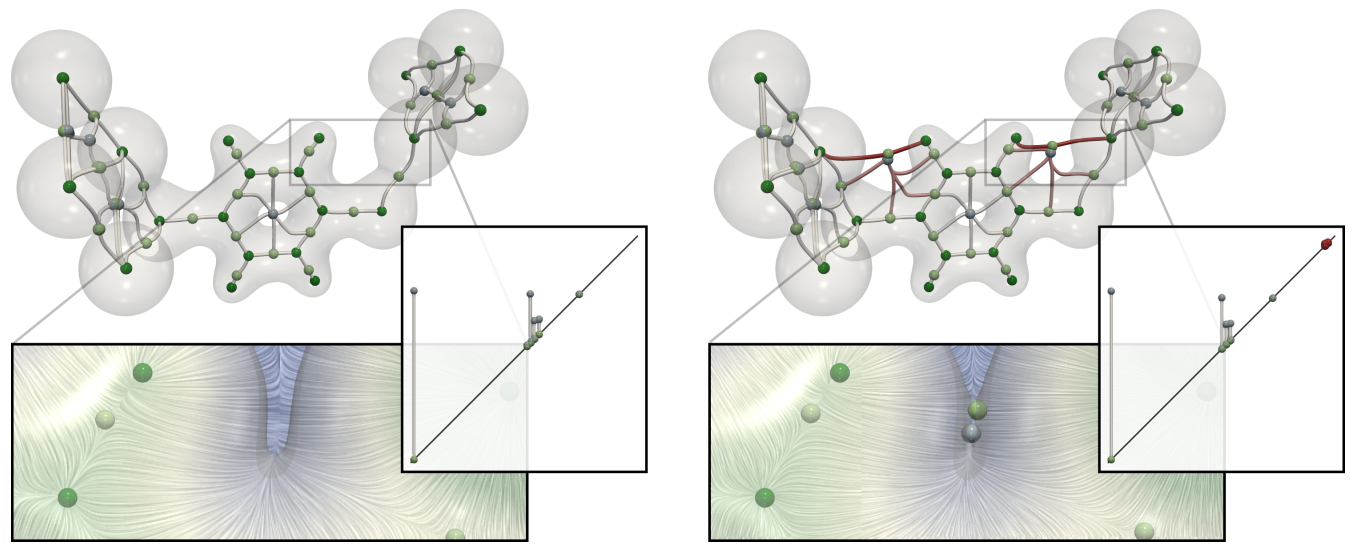

FIGURE 2 Comparison of the topological structure (one-dimensional separatrices and persistence diagram of the one-dimensional persistent homology group) for the logarithm of the electron density, $\log (\rho)$, [1] of the Au complex without (left) and with (right) relativistic effects. Isosurfaces of $\log (\rho)$ (for the same isovalues) are shown in transparent gray to illustrate the geometry of the molecule. Top: the critical points of $\log (\rho)$ are shown with colored spheres (green: maxima, light green: 2-saddles, light blue: 1-saddles) and integral lines connecting pairs of critical points are shown with white curves. With relativistic effects (right), an additional 1-saddle/2-saddle pair emerges, indicating the possible existence of a hydrogen bond (shown in dark red). Bottom: zoom on the corresponding regions (background: line integral convolution of $\nabla \log (\rho)$, colored with $\log (\rho)$ blue to green). With relativistic effects (right), the isosurface changes its genus $\left(\beta_{1}[2]\right)$ in the vicinity of the extra saddle-saddle pair (tiny handle in the surface around the pair of light green and light blue spheres). Without relativistic effects (left), the isosurface (for the same isovalue) does not exibit any handle and $\log (\rho)$ admits no saddle-saddle pair in this region of space. The persistence diagram 31 (foreground insets) of the one-dimensional persistent homology group of the sub-level sets of $\log (\rho)$ displays each saddle-saddle pair $\left(s_{1}, s_{2}\right)$ in the $2 \mathrm{D}$ plane at coordinates $\left(\log \left(\rho\left(s_{1}\right)\right), \log \left(\rho\left(s_{2}\right)\right)\right)$. The height to the diagonal $\left(\log \left(\rho\left(s_{2}\right)\right)-\log \left(\rho\left(s_{1}\right)\right)\right.$, white bar) denotes an importance measure called in Topological Data Analysis the Persistence of the critical point pair (illustrating the lifespan in the data of the corresponding topological feature). On the right diagram, the extra saddle-saddle pair is displayed in dark red (rightmost spheres). Its extremely low persistence indicates a very weak salience of the corresponding topological feature, questioning its interpretation as a hydrogen bond suggested in Anderson et al. 7 
these densities are described in detail in the caption to 2

The TDA pattern of critical points and 1-separatrices of ED is principally the same as the one obtained from QTAIM analysis. 38. However, as we shall show, TDA of ED calculated with various QM models paints a more nuanced picture.

Before continuing on that thought, we shall clarify that the presence of 1-saddles accompanying 2-saddles between $\mathrm{Au}$ and $\mathrm{H}$ atoms is a result of Morse inequalities 52 and is in accordance with chemical intuition due to the ring-shaped geometry of the Au-S-C-C-H chains. In Persistent Homology, the critical points of a scalar function are paired in a non-ambiguous way (called the Elder rule 31) and in this particular case, the additional 1-saddle and 2-saddle points, if present, form a pair on each side of the aromatic ring. The lifespan of this pair is measured by persistence, calculated as a difference of scalar field values in corresponding critical points. Accordingly, we define the persistence for the additional "BCP-RCP" pair as the difference of the (logarithm of) densities in the position of relativistic "BCPs" and "RCPs" as:2

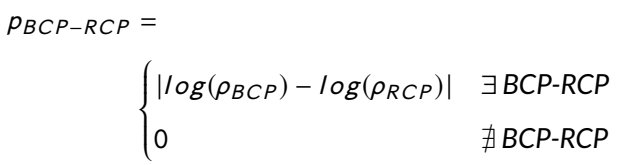

and use this definition to calculate the persistence values of extra saddle-saddle pairs of $\log (\rho)$ determined with various QM models. In all cases these values are very small, constituting typically less than $0.1 \%$ of the full persistence range (represented by red bars on the persistence diagram plotted for relativistic ED in 2]. Furthermore, they decrease with an increasing accuracy of the QM models, notably in molecules $\mathbf{g 1 ,} \mathbf{g} 2$ and g3, these persistence values systematically reduce along the DZ - TZP - QZ4P basis sets order for all XC functionals (3), a trend which is is observed for both spin states and all geometries. The most remarkable change is seen for the combination of QZ4P basis and the meta-GGA M06-2X functional, for which these saddle-saddle pairs disappear (as signified by the zero-persistence values on the plots for all but the Au4-H1 pair in the $\mathbf{g} 2$ system. This functional is also the one for which persistence values are generally lower. Taking at face value the claim of its superior performance for describing non-covalent interactions compared to the other functionals used in this work, 105, 16 our findings that this functional gives zero-persistence values would therefore imply the absence of these topological features.

At this point it is also interesting to compare results for $\mathbf{g} 2$ and $\mathrm{g} 3$, since both represent the triplet state of the $\mathrm{Au}_{4}-\mathrm{S}-\mathrm{C}_{6} \mathrm{H}_{4}-\mathrm{S}^{\prime}-\mathrm{Au}_{4}{ }_{4}$ molecule, only with a larger basis set and tighter SCF criteria used in the geometry optimization and ED calculations of the latter. It turns out that these improvements in the calculations have a tremendous effect on the persistence values of extra saddle-saddle pairs on both sides of the aromatic ring, namely these pairs are absent in $\mathbf{g} 3$ in the most accurate setups (QZ4P basis set) for all selected XC functionals. This confirms the conclusion from ref.[7] that the topology of ED significantly changes depending on how close the structure is to a minimum of the Born-Oppenheimer (BO) energy surface, but also underscores that this pair is not as significant as the conventional QTAIM analysis would make it to be.

Similar analysis of $\mathbf{m}-\mathbf{a}-\mathrm{g} 1$ and $\mathbf{m}-\mathrm{b}-\mathrm{g} 1$ 3], this time focusing on the comparison of different Hamiltonians and methods, reveals the strong influence of the electron correlation on the persistence values of the additional saddlesaddle pair, seemingly counteracting the relativistic effects. This appears from the DIRAC calculations involving the fully-relativistic 4-component DC Hamiltonian with HF and DFT/PBE methods: the ED calculated at HF level does not exhibit the extra saddle-saddle pair, while this pair is present in the DFT calculations and its persistence is (slightly) larger than when an approximate relativistic Hamiltonian (SFDC) is used. This observation, together with the negligible

\footnotetext{
${ }^{1}$ Equivalently it can be deduced from the Poincaré-Hopf theorem, which applies to generic vector fields.

2 The mathematical background of persistence can be found elsewhere. 31]
} 

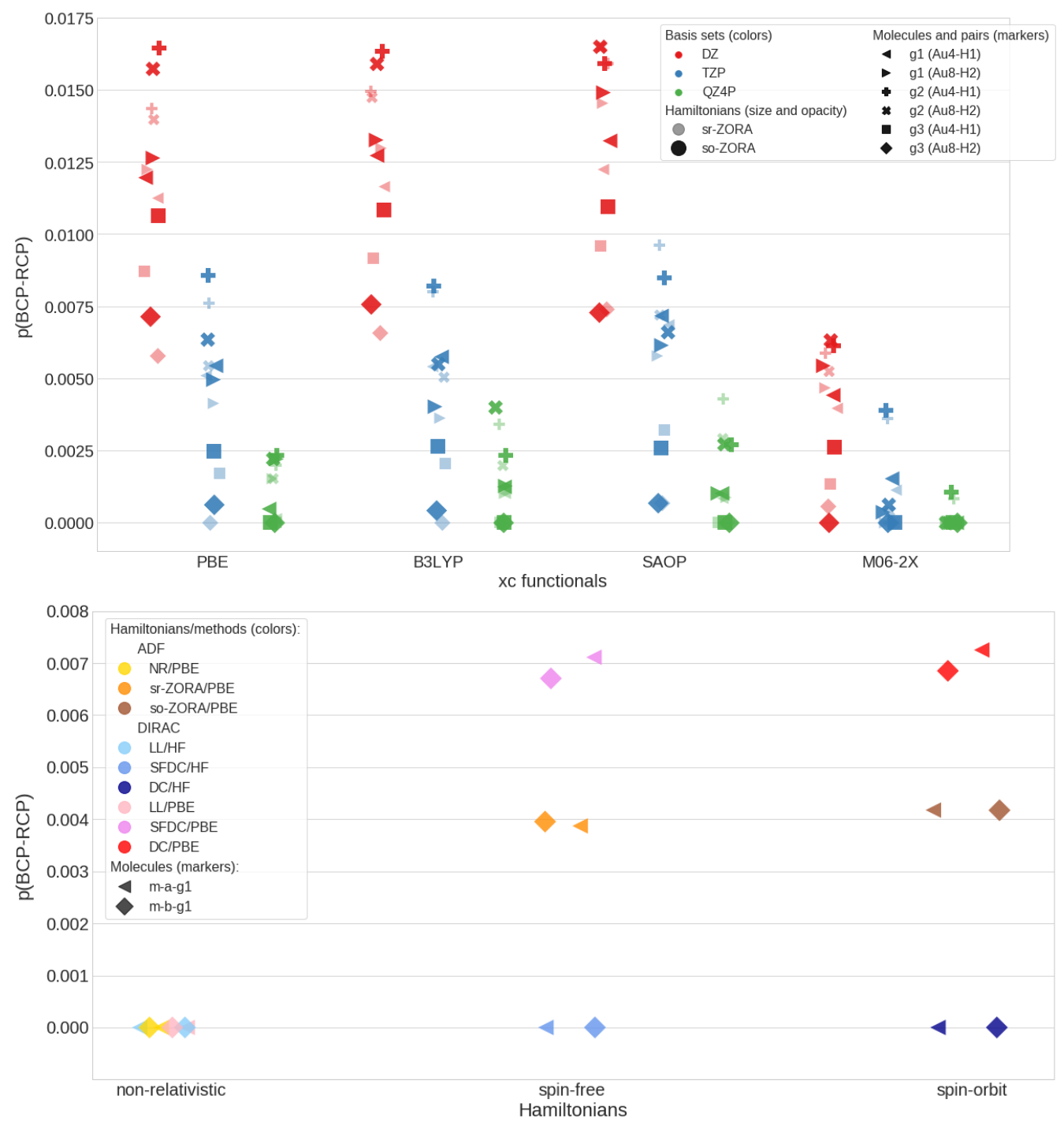

FIGURE 3 Persistence values of the extra saddle-saddle pairs of $\log (\rho)$ between $\mathrm{H} 1$ and $\mathrm{Au} 4$ and between $\mathrm{H} 2$ and Au8 atoms in $\mathrm{Au}_{4}-\mathrm{S}-\mathrm{C}_{6} \mathrm{H}_{4}-\mathrm{S}^{\prime}-\mathrm{Au}_{4}$ molecule (top) and between $\mathrm{Au}$ and $\mathrm{H}$ atoms in $\mathrm{Au}-\mathrm{S}-\mathrm{CH}-\mathrm{CH}_{2}$ molecule (bottom) calculated with different QM models. Top: the plot demonstrates that the persistence values of interesting pairs can be clustered by the basis set used in ED calculations, indicating that overall it is the basis set quality that affects the ED topology to a largest extent, while the choice of the XC functional (within tested ones), the inclusion of the spin-orbit coupling (at the ZORA level) or the chemical environment of the extra saddle-saddle pair do not have that significant effect. Bottom: the plot exposes the role of electron correlation evaluated at the DFT/PBE level in the DIRAC software and shows the differences in results obtained with ADF and DIRAC codes. The persistence values of absent saddle-saddle pairs are set to zero 2. Numerical values are available in ESI. 
role of the spin-orbit coupling on the persistence values (as evaluated on scalar-relativistic geometries), suggests further studies on $\mathbf{m}$-a-g1 and $\mathbf{m}$-b-g1 with wavefunction-based methods at the scalar-relativistic level of theory. Apart from a change in electronic structure method, it may well be that changes in equilibrium structures take place with the consideration of spin-orbit coupling in the geometry optimization procedure, as evidenced by a recent study on SOC-triggered bond contraction in gold clusters. 33] We have nevertheless opted in this work to focus on selected geometries such as those previously considered by Anderson et al. [7] This was motivated, first, by the comparison of the results for these model systems with the original molecular system, which indicates that the chemical surrounding of interacting $\mathrm{Au}$ and $\mathrm{H}$ atoms does not necessarily affect the topology of ED in these $\mathrm{Au}-\mathrm{H}$ interatomic areas. And, second, that we considered that this would divert attention to what we believe is the central point of this work, which is to underscore the differences and similarities between TDA and QTAIM. We note, however, that further studies on these gold systems should indeed consider the effect of structural chances brought about by SOC.

In TDA the consensus is that the persistence of a topological feature is associated with its importance (in particular in the presence of additive noise), therefore it is frequently used in the topological simplification of complex scalar fields. Features manifested by so small persistence values would typically be deleted, which raises the question of whether the extra "BCPs" and "RCPs" of ED are an important feature of this scalar field. The answer to this question relies on the domain knowledge to provide interpretation of such feature. In the light of late research which cast "serious doubts on the interpretation of BCPs in QTAIM analyses as indicating bonding interactions, however weak", [96] confronted with the recently proposed translation of these additional saddle-saddle pairs as indicators of "favorable interaction",[7] we are inclined to answer "no".

There are however more arguments standing for this answer, which do not involve the chemical interpretation of these additional critical points. For instance, the line integral convolution plots of the ED gradient demonstrated in 2. have a very similar pattern in relativistic and non-relativistic contexts, what is understandable considering that there need to be a border separating the atomic basins of $\mathrm{Au}$ and $\mathrm{H}$ (regardless of the QM model). In addition, as also exposed in 2 the Euclidean distance between the extra "RCP" and the extra "BCP" in relativistic case is so small, that their presence can be considered as a negligible (unimportant) perturbation to the topology of ED in this interatomic area.

In order to test the robustness of the presented analysis with respect to grid sampling, we have analyzed the topology and geometry of ED of $\mathrm{m}$-a-g1 molecule calculated with a selected QM model (so-ZORA Hamiltonian, PBE functional and three basis sets, DZ, TZP and QZ4P) on a series of regular grids of size ranging from $100^{3}$ to $320^{3}$ points. The results of this analysis are demonstrated in 4 and they confirm prior observations: the extra saddle-saddle pairs (if present) can indeed be considered as unimportant topological features of ED, if not artificial due to the expected exponential decay of the persistence values of the extra pairs of the $\rho$ scalar field with the grid size (linear dependence of the persistence values of the corresponding pairs exhibited by the $\log (\rho)$ scalar field).

What clearly discerns the relativistic ED exhibiting "BCP-RCP" pairs and nonrelativistic EDs without these pairs (for the same QM setup) is the fact that in the former case $\rho^{R}\left(r_{B C P}^{R}\right)>\rho^{R}\left(r_{R C P}^{R}\right)$, what causes the change of genus of the ED surface when sweeping over all values of ED, manifested by the appearance of additional critical points, as demonstrated in 2 In the latter case, the value of ED probed in the location of "relativistic BCP", $r_{B C P}^{R}$, is lower than the value of ED in the position of the "relativistic $R C P^{\prime}, r_{R C P}^{R}$, hence $\rho^{N R}\left(r_{B C P}^{R}\right)<\rho^{N R}\left(r_{R C P}^{R}\right)$. Consequently, the surface does not change its genus and the critical points do not appear. Yet, the difference of density values in these two locations $\left(r_{R C P}^{R}\right.$ and $\left.r_{B C P}^{R}\right)$ is very small (Table 13 in ESI), which calls for a more accurate analysis involving properties which are based on the higher-order derivatives of the ED, such as the ED gradient or the ED Hessian. [32] Other QM approximations, such as the ones determining the description of electron correlation or the quality of one-electron functions do not have such a strong influence on ED that could effectively change the total order of density values in 

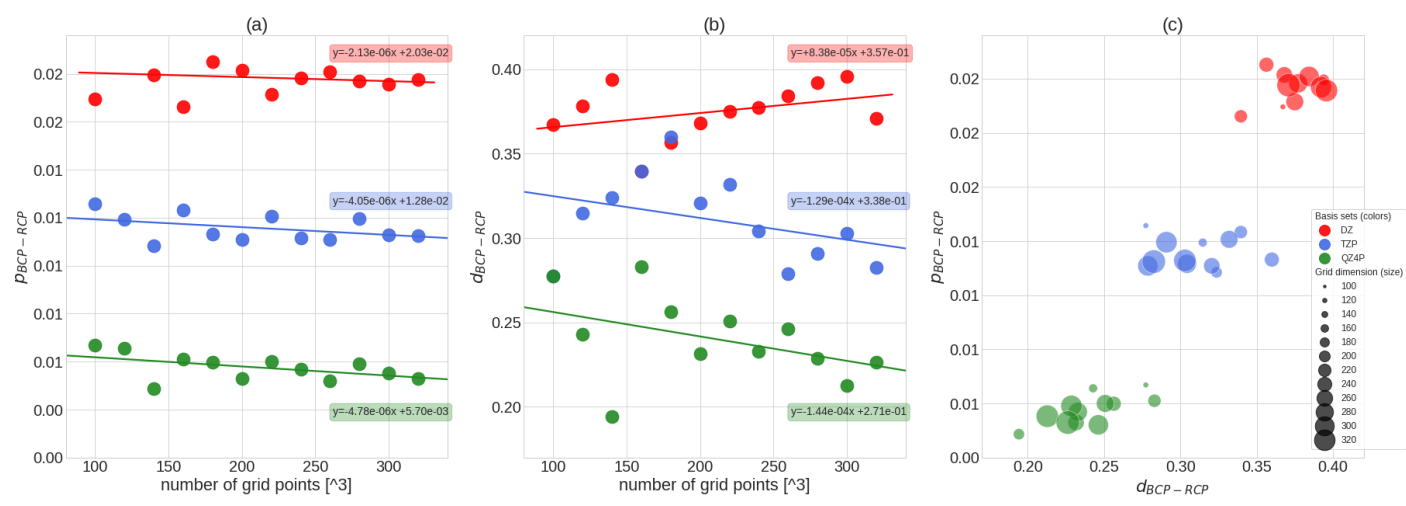

FIGURE 4 Au-S-CH-CH ${ }_{2}$ molecule (m-a-g1 model): (a) the dependence of the persistence values of the extra saddle-saddle pair of $\log (\rho)$ between $\mathrm{H}$ and $\mathrm{Au}$ atoms $\left(p_{B C P-R C P}\right)$ on the grid size, (b) the dependence of the Euclidean distance between these saddle points $\left(d_{B C P-R C P}\right)$ on the grid size and (c) the correlation of $p_{B C P-R C P}$ and $d_{B C P-R C P}$ values, with the dependence on the basis set and the grid size marked on the plot. Plots (a) and (b): the linear regression models were used to estimate a simple relationship between variables. The actual persistence values of the additional saddle-saddle pairs of $\rho$ decay exponentially with the grid size (note the $\log (r h o)$ scalar field under analysis), which suggests that the topological features under study may be artificial. The comparison of slopes of the fitted lines in DZ-TZP-QZ4P cases highlights the faster convergence of $p_{B C P-R C P}$ and $d_{B C P-R C P}$ with the grid size in the most accurate QM setup (QZ4P). Plot (c): clear data clustering with respect to basis set further emphasizes the importance of the basis set in QM-based ED topology analysis. Numerical values are available in ESI. Color coding common for all plots: DZ - red, TZP - blue, QZ4P - green markers.

these two locations, however as the final picture is a result of interplay of many effects (e.g. counteracting relativistic corrections and electron correlation), none of them should be neglected. Notably, relativistic formalism should be used if the interacting sites involve heavy elements, as already discussed elsewhere. 20 32 3

The use of accurate QM setup is essential to assert a good quality of calculated ED, however it does not change the fact that one should be cautious when drawing conclusions on bonding interactions based on the QTAIM output only. We have therefore continued with the analysis of the RDG in studied systems. As illustrated in 5 there is a striking similarity between the relativistic and non-relativistic RDGs, in geometrical (the shape of the RDG isosurfaces) and topological (the same number of connected components $\left(\beta_{0}[2]\right.$ ) and the presence and location of minima) senses. In particular, the interaction sites between $\mathrm{Au}$ and $\mathrm{H}$ atoms are present in both contexts. This is an additional argument for the resemblance of $\mathrm{Au}-\mathrm{H}$ non-covalent interactions with or without relativistic effects included.

\section{4 | CONCLUSIONS}

In conclusion, we have used TDA to reinterpret the QTAIM results for gold complexes reported recently,[7] in which relativistic effects are considered to be behind the emergence of new 1-saddle - 2-saddle point pairs between noncovalently bonded $\mathrm{Au}$ and $\mathrm{H}$ atoms.

The choice of TDA is motivated by its favorable performance (by using combinatorial algorithms instead of numerical grid searches) and mathematical robustness, allowing for an explicit translation between topological features and their chemical interpretation. TDA offers the possibility of visualizing the "topological skeleton" of a scalar field, including for the EDs the same features as QTAIM (critical points and 1-separatrices between 2-saddles and maxima that correspond 

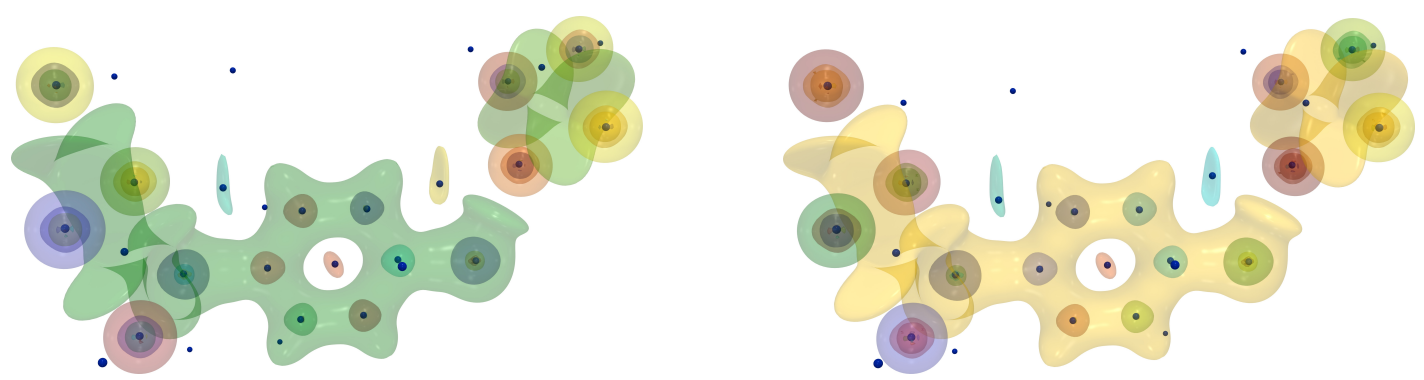

FIGURE 5 Comparison of data segmentations based on isosurfaces of reduced density gradient (same isovalue) without (left) and with (right) relativistic effects for the Au complex. Each connected component of isosurface is displayed with a unique, randomly chosen, color. The number of connected components is equal in the two cases, and their geometry is highly similar. In particular, the regions exhibiting extra saddle-saddle pairs of $\log (\rho)$ [2] contain isosurface components with very similar geometry in both cases.

to "bond paths") as well as others that do not have their QTAIM correspondence (1-separatrices between 1-saddles and 2-saddles, 2-separatrices, manifolds of the Morse-Smale complex and outputs from other TDA data abstractions).

Our results demonstrate that the relationship between the topology of ED and the existence of non-covalent interactions is more complex than what can be deduced from a QTAIM picture based on the presence or absence of "BCPs" and "bond paths" between atoms. While TDA confirms that the additional saddle-saddle pairs between Au and $\mathrm{H}$ atoms are indeed the features of the relativistic ED which are absent for a non-relativistic ED, these feature appear not to be meaningful, due to their low persistence values and, more importantly, to the fact that these values approach zero with increased sophistication of the calculations (larger basis set and denser grids).

Furthermore, our observations are another example of the by now established fact that the ED is not the best descriptor of such weak interactions, for which other quantities such as the RDG are better suited. 46. 23. 17. 57. Our geometrical and topological analysis of the RDG, however, does not show qualitative differences between the non-relativistic and relativistic calculations, a fact which further calls into question the importance of relativistic effects to non-covalent interactions in such systems.

Taken together, these conclusions point to the need for a deeper analysis of the influence of the relativistic effects on the non-covalent interactions. To this end, we have shown here that much smaller model systems retain the same topological features as the original molecules, paving the way for more accurate calculations, such as those based on correlated wavefunction methods.

Finally, we suggest that TDA, and notably the concept of persistence-which can be considered as a process of transforming discrete invariant into a continuous one [5]-should be considered in the development of real-space analysis techniques of the chemically-relevant scalar fields.

\section{ACKNOWLEDGEMENTS}

We acknowledge support from the Polish National Science Centre (NCN) (grant number 2016/23/D/ST4/03217), the Labex CaPPA (contract ANR-11-LABX-0005-01) and I-SITE ULNE project OVERSEE (contract ANR-16-IDEX0004), CPER CLIMIBIO (European Regional Development Fund, Hauts de France council, French Ministry of Higher Education and Research), the French national supercomputing facilities (grant DARI A0050801859), and the European Commission grant H2020-FETHPC-2017 “VESTEC” (ref. 800904). We would like to thank Dr Valérie Vallet for her comments on the draft of this paper. 


\section{CONFLICT OF INTEREST}

There are no conflicts to declare.

\section{SUPPORTING INFORMATION AVAILABLE:}

The supporting information is available on zenodo, DOI:10.5281/zenodo.3358788.

\section{REFERENCES}

[1] The logarithm scale was employed in the TDA of ED since the full persistence range calculated for the ED is very large, what makes the visualization of bars on the persistence diagram corresponding to the extra saddle-saddle pairs in its topology (exhibiting very low persistence values) impractical. The logarithm scale for the RDG was then used for consistency.

[2] Betti numbers, $\beta_{0}$ and $\beta_{1}$, signify the number of connected components and the number of one-dimensional holes, respectively, https://en.wikipedia.org/wiki/Betti_number

[3] DIRAC, a relativistic ab initio electronic structure program, Release DIRAC18 (2018), written by T. Saue, L. Visscher, H. J. Aa. Jensen, and R. Bast, with contributions from V. Bakken, K. G. Dyall, S. Dubillard, U. Ekström, E. Eliav, T. Enevoldsen, E. Faßhauer, T. Fleig, O. Fossgaard, A. S. P. Gomes, E. D. Hedegård, T. Helgaker, J. Henriksson, M. Iliaš, Ch. R. Jacob, S. Knecht, S. Komorovský, O. Kullie, J. K. Lærdahl, C. V. Larsen, Y. S. Lee, H. S. Nataraj, M. K. Nayak, P. Norman, G. Olejniczak, J. Olsen, J. M. H. Olsen, Y. C. Park, J. K. Pedersen, M. Pernpointner, R. di Remigio, K. Ruud, P. Sałek, B. Schimmelpfennig, A. Shee, J. Sikkema, A. J. Thorvaldsen, J. Thyssen, J. van Stralen, S. Villaume, O. Visser, T. Winther, and S. Yamamoto (available at https://doi.org/10.5281/zenodo.2253986 see also http://www.diracprogram. org).

[4] Basis set (dyall.acv3z) available from the Dirac web site, http://dirac.chem.sdu.dk/basisarchives/dyall/index. html.

[5] Wojciech Chachólski, 'How to give a machine a sense of geometry?', keynote talk in TopolnVis 2019, June 17, 2019 , Nykoping, Sweden.

[6] James S. M. Anderson, Juan I. Rodríguez, Paul W. Ayers, and Andreas W. Götz. Relativistic (SR-ZORA) quantum theory of atoms in molecules properties. J. Comput. Chem., 38(2):81-86, jan 2017.

[7] James S.M. Anderson, Juan I. Rodríguez, Paul W. Ayers, Daniel E. Trujillo-González, Andreas W. Götz, Jochen Autschbach, Fray L. Castillo-Alvarado, and Koichi Yamashita. Molecular QTAIM Topology Is Sensitive to Relativistic Corrections. Chem. Eur. J., 25:2538-2544, jan 2019.

[8] Paul W. Ayers, Russell J. Boyd, Patrick Bultinck, Michel Caffarel, Ramon Carbó-Dorca, Mauro Causá, Jerzy Cioslowski, Julia Contreras-García, David L. Cooper, Philip Coppens, Carlo Gatti, Simon Grabowsky, Paolo Lazzeretti, Piero Macchi, Ángel Martín Pendás, Paul L. A. Popelier, Klaus Ruedenberg, Henry Rzepa, Andreas Savin, Alexander Sax, W.H. Eugen Schwarz, Shant Shahbazian, Bernard Silvi, Miquel Solà, and Vladimir Tsirelson. Six questions on topology in theoretical chemistry. Comput. Theor. Chem., 1053:2-16, feb 2015.

[9] R. F.W. Bader. A bond path: A universal indicator of bonded interactions. J. Phys. Chem. A, 102(37):7314-7323, 1998.

[10] Richard F. W. Bader. Atoms in Molecules. International Series of Monographs on Chemistry. Clarendon Press, 1994.

[11] Richard F.W. Bader. A quantum theory of molecular structure and its applications. Chem. Rev., 91(5):893-928, jul 1991.

[12] Richard F.W. Bader. Bond paths are not chemical bonds. J. Phys. Chem. A, 113(38):10391-10396, sep 2009. 
[13] Evert Jan Baerends, Tom Ziegler, A. J. Atkins, Jochen Autschbach, Donald Bashford, O. Baseggio, A. Bérces, F. Matthias Bickelhaupt, C. Bo, P. M. Boerritger, Luigi Cavallo, C. Daul, D. P. Chong, D. V. Chulhai, L. Deng, R. M. Dickson, J. M. Dieterich, D. E. Ellis, M. van Faassen, A. Ghysels, A. Giammona, Stan J. A. van Gisbergen, A. Goez, A. W. Götz, S. Gusarov, F. E. Harris, P. van den Hoek, Z. Hu, Christoph R. Jacob, H. Jacobsen, L. Jensen, L. Joubert, J. W. Kaminski, G. van Kessel, C. König, F. Kootstra, A. Kovalenko, Mykhaylo Krykunov, Erik van Lenthe, D. A. McCormack, A. Michalak, M. Mitoraj, S. M. Morton, Johannes Neugebauer, V. P. Nicu, Louis Noodleman, V. P. Osinga, S. Patchkovskii, Michele Pavanello, C. A. Peeples, Pierre Herman Theodoor Philipsen, D. Post, Cory C. Pye, H. Ramanantoanina, P. Ramos, W. Ravenek, J. I. Rodríguez, P. Ros, R. Rüger, P. R. T. Schipper, D. Schlüns, H. van Schoot, G. Schreckenbach, J. S. Seldenthuis, Mike Seth, Jaap G. Snijders, Miquel Solà, Stener M., M. Swart, D. Swerhone, G. te Velde, V. Tognetti, P. Vernooijs, L. Versluis, Lucas Visscher, O. Visser, Fan Wang, T. A. Wesolowski, E. M. van Wezenbeek, G. Wiesenekker, S. K. Wolff, T. K. Woo, and A. L. Yakovlev. ADF2017, SCM, Theoretical Chemistry, Vrije Universiteit, Amsterdam, The Netherlands, https://www.scm.com.

[14] K. Beketayev, G. H. Weber, M. Haranczyk, P. T. Bremer, M. Hlawitschka, and B. Hamann. Topology-based visualization of transformation pathways in complex chemical systems. COMPUT GRAPH FORUM, 30(3):663-672, jun 2011.

[15] Harsh Bhatia, Attila G. Gyulassy, Vincenzo Lordi, John E. Pask, Valerio Pascucci, and Peer-Timo Bremer. T opo MS : Comprehensive topological exploration for molecular and condensed-matter systems. J. Comput. Chem., 39:936, mar 2018.

[16] Martin P. Bircher, Pablo López-Tarifa, and Ursula Rothlisberger. Shedding Light on the Basis Set Dependence of the Minnesota Functionals: Differences between Plane Waves, Slater Functions, and Gaussians. J. Chem. Theory Comput., 15(1):557-571, jan 2019.

[17] Roberto A. Boto, Jean Philip Piquemal, and Julia Contreras-García. Revealing strong interactions with the reduced density gradient: a benchmark for covalent, ionic and charge-shift bonds. Theor. Chem. Acc., 136(12):139, dec 2017.

[18] Didier Bourissou. Changing the gold standard. Nat. Chem., 11(3):199-200, mar 2019.

[19] David Brown. Another look at bonds and bonding. Structural Chemistry, oct 2019.

[20] Lukáš Bučinský, Dylan Jayatilaka, and Simon Grabowsky. Importance of relativistic effects and electron correlation in structure factors and electron density of diphenyl mercury and triphenyl bismuth. J. Phys. Chem. A, 120(33):6650-6669, aug 2016.

[21] Erick Cerpa, Andreas Krapp, Alberto Vela, and Gabriel Merino. The Implications of Symmetry of the External Potential on Bond Paths. Chem. Eur. J., 14(33):10232-10234, nov 2008.

[22] Timothy Clark, Jane S. Murray, and Peter Politzer. A perspective on quantum mechanics and chemical concepts in describing noncovalent interactions. Phys. Chem. Chem. Phys., 20(48):30076-30082, dec 2018.

[23] Julia Contreras-García, Roberto A. Boto, Fernando Izquierdo-Ruiz, Igor Reva, Tatiana Woller, and Mercedes Alonso. A benchmark for the non-covalent interaction $(\mathrm{NCl})$ index or... is it really all in the geometry? Theor. Chem. Acc., 135(10):242, oct 2016.

[24] Julia Contreras-García, Erin R. Johnson, Shahar Keinan, Robin Chaudret, Jean Philip Piquemal, David N. Beratan, and Weitao Yang. NCIPLOT: A program for plotting noncovalent interaction regions. J. Chem. Theory Comput., 7(3):625-632, mar 2011.

[25] Jurgens H. de Lange, Daniël M. E. van Niekerk, and Ignacy Cukrowski. Quantifying individual (anti)bonding molecular orbitals' contributions to chemical bonding. Phys. Chem. Chem. Phys., 21(37):20988-20998, 2019.

[26] Jean Pierre Dognon. Electronic structure theory to decipher the chemical bonding in actinide systems. Coord. Chem. Rev., 344:150-162, aug 2017.

[27] K. G. Dyall and K. Jr Faegri. Introduction to Relativistic Quantum Chemistry. Oxfrod University Press, 2007. 
[28] Kenneth G. Dyall. An exact separation of the spin-free and spin-dependent terms of the Dirac-Coulomb-Breit Hamiltonian. J. Chem. Phys., 100(3):2118-2127, feb 1994.

[29] Kenneth G. Dyall. Relativistic double-zeta, triple-zeta, and quadruple-zeta basis sets for the $6 \mathrm{~d}$ elements Rf-Cn. Theor. Chem. Acc., 129(3-5):603-613, jun 2011.

[30] Kenneth G. Dyall. Core correlating basis functions for elements 31-118. Theor. Chem. Acc., 131(5):1217, may 2012.

[31] H. Edelsbrunner and J. Harer. Computational Topology: An Introduction. American Mathematical Society, 2009.

[32] Georg Eickerling, Remigius Mastalerz, Verena Herz, Wolfgang Scherer, Hans Jörg Himmel, and Markus Reiher. Relativistic effects on the topology of the electron density. J. Chem. Theory Comput., 3(6):2182-2197, 2007.

[33] Mauricio A. Flores and Eduardo Menendez-Proupin. Spin-orbit coupling effects in gold clusters: The case of $\mathrm{Au}_{13}$. In J. Phys.: Conf. Ser., volume 720, page 012034, 2016.

[34] C. Fonseca Guerra, J. G. Snijders, G. Te Velde, and E. J. Baerends. Towards an order-N DFT method. Theor. Chem. Acc., 99(6):391-403, nov 1998.

[35] Cina Foroutan-Nejad, Shant Shahbazian, and Radek Marek. Toward a consistent interpretation of the QTAIM: Tortuous link between chemical bonds, interactions, and bond/line paths. Chem. Eur. J., 20(32):10140-10152, aug 2014.

[36] Carlo Gatti, Piercarlo Fantucci, and Gianfranco Pacchioni. Charge density topological study of bonding in lithium clusters. Theor. Chim. Acta, 72(5-6):433-458, dec 1987.

[37] O. V. Gritsenko, P. R.T. Schipper, and E. J. Baerends. Approximation of the exchange-correlation Kohn-Sham potential with a statistical average of different orbital model potentials. Chem. Phys. Lett., 302(3-4):199-207, mar 1999.

[38] David Günther, Roberto A. Boto, Julia Contreras-García, Jean-Philip Piquemal, and Julien Tierny. Characterizing molecular interactions in chemical systems. IEEE Trans. Vis. Comput. Graph., 20(12):2476-2485, dec 2014.

[39] Hannu Häkkinen. The gold-sulfur interface at the nanoscale. Nat. Chem., 4(6):443-455, jun 2012.

[40] W. J. Hehre, L. Radom, P. v. R. Schleyer, and J. A. Pople. Ab Initio Molecular Orbital Theory. Wiley, New York, 1986.

[41] C. Heine, H. Leitte, M. Hlawitschka, F. luricich, L. De Floriani, G. Scheuermann, H. Hagen, and C. Garth. A Survey of Topology-based Methods in Visualization. COMPUT GRAPH FORUM, 35:643, 2016.

[42] Pedro Hermosilla, Jorge Estrada, Victor Guallar, Timo Ropinski, Àlvar Vinacua, and Pere-Pau Vázquez. Physics-Based Visual Characterization of Molecular Interaction Forces. IEEE Trans. Vis. Comput. Graph., 23(1):731-740, jan 2017.

[43] P. Hohenberg and W. Kohn. Inhomogeneous electron gas. Phys. Rev., 136(3B):B864-B871, nov 1964.

[44] Mirosław Jabłoński. On the Uselessness of Bond Paths Linking Distant Atoms and on the Violation of the Concept of Privileged Exchange Channels. ChemistryOpen, 8(4):497-507, apr 2019.

[45] Erin R. Johnson, Shahar Keinan, Paula Mori-Sánchez, Julia Contreras-García, Aron J. Cohen, and Weitao Yang. Revealing noncovalent interactions. J. Am. Chem. Soc., 132(18):6498-6506, may 2010.

[46] Joseph R. Lane, Julia Contreras-García, Jean-Philip Piquemal, Benjamin J. Miller, and Henrik G. Kjaergaard. Are bond critical points really critical for hydrogen bonding? J. Chem. Theory Comput., 9(8):3263-3266, aug 2013.

[47] Jean-Marc Lévy-Leblond. Nonrelativistic particles and wave equations. Commun. Math. Phys., 6(4):286-311, dec 1967.

[48] Dan R. Lipşa, Robert S. Laramee, Simon J. Cox, Jonathan C. Roberts, Rick Walker, Michelle A. Borkin, and Hanspeter Pfister. Visualization for the physical sciences. COMPUT GRAPH FORUM, 31(8):2317-2347, dec 2012. 
[49] Stefan Mebs, Roman Kalinowski, Simon Grabowsky, Diana Förster, Rainer Kickbusch, Eugen Justus, Wolfgang Morgenroth, Carsten Paulmann, Peter Luger, Detlef Gabel, and Dieter Lentz. Real-space indicators for chemical bonding. Experimental and theoretical electron density studies of four deltahedral boranes. Inorg. Chem., 50(1):90-103, jan 2011.

[50] Ingrid Membrillo-Solis, Mariam Pirashvili, Lee Steinberg, Jacek Brodzki, and Jeremy G Frey. Topology and geometry of molecular conformational spaces and energy landscapes. 2019.

[51] Paul G. Mezey. The Holographic Electron Density Theorem, de-quantization, re-quantization, and nuclear charge space extrapolations of the Universal Molecule Model. In AIP Conference Proceedings, volume 1906, page 020001. AIP Publishing LLC, nov 2017.

[52] J. Milnor. Morse Theory. Princeton U. Press, 1963.

[53] Christophe Narth, Zeina Maroun, Roberto A. Boto, Robin Chaudret, Marie-Laure Bonnet, Jean-Philip Piquemal, and Julia Contreras-García. A Complete NCI Perspective: From New Bonds to Reactivity. In Applications of Topological Methods in Molecular Chemistry, pages 491-527. Springer International Publishing, Cham, 2016.

[54] Alberto Otero-De-La-Roza, Erin R. Johnson, and Julia Contreras-García. Revealing non-covalent interactions in solids: NCl plots revisited. Phys. Chem. Chem. Phys., 14(35):12165-12172, aug 2012.

[55] Nina Otter, Mason A. Porter, Ulrike Tillmann, Peter Grindrod, and Heather A. Harrington. A roadmap for the computation of persistent homology. EPJ Data Science, 6(1):17, dec 2017.

[56] Ewa Pastorczak and Clémence Corminboeuf. Perspective: Found in translation: Quantum chemical tools for grasping non-covalent interactions. J. Chem. Phys., 146(12):120901, mar 2017.

[57] Francesca Peccati, Eline Desmedt, and Julia Contreras-García. A regression approach to accurate interaction energies using topological descriptors. Comput. Theor. Chem., 1159:23-26, jul 2019.

[58] Evangelina Pensa, Emiliano Cortés, Gastón Corthey, Pilar Carro, Carolina Vericat, Mariano H. Fonticelli, Guillermo Benítez, Aldo A. Rubert, and Roberto C. Salvarezza. The chemistry of the sulfur-gold interface: In search of a unified model. Acc. Chem. Res., 45(8):1183-1192, aug 2012.

[59] John P. Perdew, Kieron Burke, and Matthias Ernzerhof. Generalized gradient approximation made simple. Phys. Rev. Lett., 77(18):3865-3868, oct 1996.

[60] Julien Pilmé, Eric Renault, Fadel Bassal, Mohamed Amaouch, Gilles Montavon, and Nicolas Galland. QTAIM analysis in the context of quasirelativistic quantum calculations. J. Chem. Theory Comput., 10(11):4830-4841, nov 2014.

[61] Mariam Pirashvili, Lee Steinberg, Francisco Belchi Guillamon, Mahesan Niranjan, Jeremy G. Frey, and Jacek Brodzki. Improved understanding of aqueous solubility modeling through topological data analysis. J. Cheminformatics, 10(1):54, dec 2018.

[62] Peter Politzer and Jane S. Murray. A look at bonds and bonding. STRUCT CHEM, 30:1153-1157, jun 2019.

[63] Robert Ponec and Carlo Gatti. Do the structural changes defined by the electron density topology necessarily affect the picture of the bonding? Inorg. Chem., 48(23):11024-11031, dec 2009.

[64] Paul Lode Albert Popelier. The QTAIM Perspective of Chemical Bonding, chapter 8, pages 271-308. John Wiley \& Sons, Ltd, 2014.

[65] John A. Pople. Nobel Lecture: Quantum chemical models. Rev. Mod. Phys., 71(5):1267-1274, oct 1999.

[66] Pekka Pyykkö. Theoretical Chemistry of Gold. Angew. Chem. Int. Ed., 43(34):4412-4456, aug 2004.

[67] Pekka Pyykkö. Theoretical chemistry of gold. II. Inorg. Chim. Acta, 358(14):4113-4130, nov 2005. 
[68] Pekka Pyykkö. Theoretical chemistry of gold. III. Chem. Soc. Rev., 37(9):1967, aug 2008.

[69] Pekka Pyykkö. Relativistic Effects in Chemistry: More Common Than You Thought. Annu. Rev. Phys. Chem., 63(1):45-64, may 2012.

[70] M. Reiher and A. Wolf. Relativistic Quantum Chemistry. The Fundamental Theory of Molecular Science. WILEY-VCH, 2009.

[71] Mathilde Rigoulet, Stéphane Massou, E Daiann Sosa Carrizo, Sonia Mallet-Ladeira, Abderrahmane Amgoune, Karinne Miqueu, and Didier Bourissou. Evidence for genuine hydrogen bonding in gold(I) complexes. Proc. Natl. Acad. Sci. U.S.A., 116(1):46-51, jan 2018.

[72] Juan I. Rodríguez, Emilbus A. Uribe, María I. Baltazar-Méndez, Jochen Autschbach, F. L. Castillo-Alvarado, and Israel Gutiérrez-González. Size evolution relativistic DFT-QTAIM study on the gold cluster complexes Au4-S-CnH2n-S"-Au4" ( $\mathrm{n}=2-5)$. Chem. Phys. Lett., 660:287-294, sep 2016.

[73] Juan I. Rodríguez, Ulises A. Vergara-Beltran, and Jochen Autschbach. Size evolution study on the electronic and optical properties of gold-cluster complexes $\mathrm{Au}_{4}-\mathrm{S}-\mathrm{C}_{n}-\mathrm{H}_{2 n}-\mathrm{S}^{\prime}-\mathrm{Au}_{4}{ }^{\prime}(\mathrm{n}=2-5)$. Chem. Phys. Lett., 732:136625, oct 2019.

[74] Trond Saue. Relativistic Hamiltonians for chemistry: a primer. ChemPhysChem, 12(17):3077-94, dec 2011.

[75] P. R.T. Schipper, O. V. Gritsenko, S. J.A. Van Gisbergen, and E. J. Baerends. Molecular calculations of excitation energies and (hyper)polarizabilities with a statistical average of orbital model exchange-correlation potentials. J. Chem. Phys., 112(3):1344-1352, jan 2000.

[76] Hubert Schmidbaur. Proof of Concept for Hydrogen Bonding to Gold, Au . . H-X. Angew. Chem. Int. Ed., 58(18):58065809 , apr 2019.

[77] Hubert Schmidbaur, Helgard G. Raubenheimer, and Liliana Dobrzańska. The gold-hydrogen bond, Au-H, and the hydrogen bond to gold, Au . . H-X. Chem. Soc. Rev., 43(1):345-380, dec 2014.

[78] P. Schwerdtfeger. Relativistic Electronic Structure Theory. Part 1. Fundamentals. In Theoretical and Computational Chemistry, volume 11, pages 1-926. Elsevier B.V., 2002.

[79] P. Schwerdtfeger. Relativistic Electronic Structure Theory. Part 2. Applications. In Theoretical and Computational Chemistry, volume 14, pages 1-787. Elsevier B.V., 2004.

[80] Shant Shahbazian. Why Bond Critical Points Are Not "Bond" Critical Points. Chem. Eur. J., 24(21):5401-5405, apr 2018.

[81] Lee Steinberg, John Russo, and Jeremy Frey. A new topological descriptor for water network structure. J. Cheminformatics, 11(1):48, dec 2019. Correction: ibid. 11, 51 (2019).

[82] P. J. Stephens, F. J. Devlin, C. F. Chabalowski, and M. J. Frisch. Ab Initio calculation of vibrational absorption and circular dichroism spectra using density functional force fields. J. Phys. Chem., 98(45):11623-11627, nov 1994.

[83] Michal Straka, Erik Andris, Jan Vícha, Aleš Rǔžička, Jana Roithová, and Lubomír Rulíšek. Spectroscopic and Computational Evidence of Intramolecular Au ${ }^{I} \ldots \mathrm{H}^{+}-\mathrm{N}$ Hydrogen Bonding. Angew. Chem. Int. Ed., 58(7):2011-2016, feb 2019.

[84] G. te Velde, F. M. Bickelhaupt, E. J. Baerends, C. Fonseca Guerra, S. J. A. van Gisbergen, J. G. Snijders, and T. Ziegler. Chemistry with ADF. J. Comput. Chem., 22(9):931-967, 2001.

[85] J. Tierny. Topological Data Analysis for Scientific Visualization. In Mathematics and Statistics. Springer, Cham, 2017.

[86] Julien Tierny, Guillaume Favelier, Joshua A. Levine, Charles Gueunet, and Michael Michaux. The Topology ToolKit. IEEE Trans. Vis. Comput. Graph. (Proc. of IEEE VIS), 24:832-842, 2017. https : //topology-tool-kit.github.io/.

[87] Chao Tong, Richard Roberts, Rita Borgo, Sean Walton, Robert S. Laramee, Kodzo Wegba, Aidong Lu, Yun Wang, Huamin Qu, Qiong Luo, and Xiaojuan Ma. Storytelling and visualization: An extended survey. Information (Switzerland), 9(3):65, mar 2018. 
[88] E. Van Lenthe and E. J. Baerends. Optimized Slater-type basis sets for the elements 1-118. J. Comput. Chem., 24(9):1142-1156, jul 2003.

[89] E. Van Lenthe, E. J. Baerends, and J. G. Snijders. Relativistic total energy using regular approximations. J. Chem. Phys., 101(11):9783-9792, dec 1994.

[90] E. Van Lenthe, J. G. Snijders, and E. J. Baerends. The zero-order regular approximation for relativistic effects: The effect of spin-orbit coupling in closed shell molecules. J. Chem. Phys., 105(15):6505-6516, oct 1996.

[91] E. Van Lenthe, R. Van Leeuwen, E. J. Baerends, and J. G. Snijders. Relativistic regular two-component hamiltonians. Int. J. Quant. Chem., 57(3):281-293, sep 1996.

[92] Erik Van Lenthe. Geometry optimizations in the zero order regular approximation for relativistic effects. J. Chem. Phys., 110(18):8943-8953, may 1999.

[93] Sanjay K. Verma, Shagufi Naz Ansari, Pratibha Kumari, and Shaikh M. Mobin. Click Reaction Driven, Highly Fluorescent Dinuclear Organogold(I) Complex Exhibits a Dual Role: A Rare Au...H Interaction and an Antiproliferative Agent. Organometallics, 38:2591, jul 2019.

[94] Lucas Visscher. Approximate molecular relativistic Dirac-Coulomb calculations using a simple Coulombic correction. Theor. Chem. Acc., 98(2-3):68-70, dec 1997.

[95] Gunther H. Weber, Scott E. Dillard, Hamish Carr, Valerio Pascucci, and Bernd Hamann. Topology-controlled volume rendering. IEEE Trans. Vis. Comput. Graph., 13(2):330-341, mar 2007.

[96] Christian R. Wick and Timothy Clark. On bond-critical points in QTAIM and weak interactions. J. Mol. Model., 24(6):142, jun 2018.

[97] Timothy R. Wilson and M.E. Eberhart. Quantum theory of atoms in molecules in condensed charge density space. Can. J. Chem., pages 1-6, jul 2019.

[98] David E. Woon and Thom H. Dunning. Gaussian basis sets for use in correlated molecular calculations. iii. the atoms aluminum through argon. J. Chem. Phys., 98:1358-1371, 1993.

[99] Kedi Wu, Zhixiong Zhao, Renxiao Wang, and Guo Wei Wei. TopP-S: Persistent homology-based multi-task deep neural networks for simultaneous predictions of partition coefficient and aqueous solubility. J. Comput. Chem., 39(20):14441454, jul 2018.

[100] Kelin Xia. Persistent homology analysis of ion aggregations and hydrogen-bonding networks. Phys. Chem. Chem. Phys., 20(19):13448-13460, may 2018.

[101] Kelin Xia, D. Vijay Anand, Saxena Shikhar, and Yuguang Mu. Persistent homology analysis of osmolyte molecular aggregation and their hydrogen-bonding networks. Phys. Chem. Chem. Phys., 21(37):21038-21048, 2019.

[102] Kelin Xia, Xin Feng, Yiying Tong, and Guo Wei Wei. Persistent homology for the quantitative prediction of fullerene stability. J. Comput. Chem., 36(6):408-422, mar 2015.

[103] Yurui Xue, Xun Li, Hongbin Li, and Wenke Zhang. Quantifying thiol-gold interactions towards the efficient strength control. Nat. Commun., 5(1):4348, dec 2014.

[104] Yan Zhao and Donald G. Truhlar. A new local density functional for main-group thermochemistry, transition metal bonding, thermochemical kinetics, and noncovalent interactions. J. Chem. Phys., 125(19):194101, nov 2006.

[105] Yan Zhao and Donald G. Truhlar. The M06 suite of density functionals for main group thermochemistry, thermochemical kinetics, noncovalent interactions, excited states, and transition elements: Two new functionals and systematic testing of four M06-class functionals and 12 other function. Theor. Chem. Acc., 120(1-3):215-241, may 2008. 